\title{
Assessment of Ecological Responses to Environmental Flow Regimes using a Decision Support System Framework
}

\author{
Booty, W.G. ${ }^{l}$, Young, W.J. ${ }^{2}$, Lam, D.C.L. ${ }^{\prime}$, Whigham, P.A. ${ }^{2}$, Wong, I.W.S. ${ }^{l}$, \\ Cuddy, S.M. ${ }^{2}$, Brown, K. $^{3}$ and Farley, T.F. ${ }^{2}$ \\ ${ }^{I}$ National Water Research Institute, 867 Lakeshore Rd, Burlington, Ont. Canada, \\ ${ }^{2}$ CSIRO Land and Water, GPO Box 1666, ACT 2601 Australia \\ ${ }^{3}$ ES Aquatic, Inc., Kitchener, Ont. Canada
}

Key words: Ecological river modelling, decision support system, environmental assessment.

Abstract The Environmental Flows Decision Support System (EFDSS) has been developed to allow the communities and governments in the Murray-Darling Basin of Australia to assess the environmental responses of the lowland floodplain rivers to proposed flow management scenarios. The system integrates a range of qualitative and quantitative ecological models which consider blooms of toxic blue-green algae as well as habitat conditions for fish, floodplain vegetation, and waterbird breeding. It has been designed to accommodate a wide range of users. In this paper the overall design concepts and components of the EFDSS are described.

\section{INTRODUCTION}

In Australia, one of the most important environmental problems is the management of the rivers to arrive at an acceptable balance between competing human and natural uses. For example, in the Murray-Darling Basin, excessive water diversion, consumption, and highly modified flow regimes due to regulation have caused significant ecological impacts. In order to assist the community and government in making informed trade-off decisions, an environmental flows decision support system (EFDSS) has been developed to provide scientifically-based predictions of the likely 
responses of the river ecosystem components to proposed flow management scenarios. The development of the EFDSS represents a collaborative effort between the Commonwealth Science and Industry Research Organization (CSIRO) and the National Water Research Institute (NWRI). The system has been developed using tools from the RAISON for Windows Decision Support System (Lam et al. 1994) which has been developed at NWRI over the last 13 years. It has been designed to be able to operate in a community context and has been developed and tested using real data and public feedback during all stages of development for the Borders River catchment of New South Wales and Queensland.

In the following sections we present an overview and rationale of the EFDSS structure as well as the models developed and their integration through both technical and public user interfaces.

\section{SYSTEM DESIGN}

The overall system design of EFDSS was derived after several iterations taking into consideration available data- and knowledge-bases. It was also based on feedback from meetings with communities in the Border River catchment area. Because of the strong requirements to meet the purposes of public consultation, the separation of a public user interface from the technical user interface is essential (Young et al., 1997). These two interfaces are mainly linked through databases and data loaders (an interface to convert external data into the EFDDS database). The use of the database approach is also to ensure portability from one location to another, as new data and model results can be added via databases.

The main source of flow information came from the IQQM (Integrated Quantity Quality Model, Podger et al., 1994) which operates externally from the EFDSS. This model is used by resource management agencies to produce hydrologic flow scenarios. Daily flow data for each locality and locality type (river section, weir pool, floodplain, wetland, billabong, and lake) are provided at selected IQQM nodes, typically for a time span of one hundred years. Outputs from the model are used as direct input to the floodplain model and ecology-hydrology indices model, which operate within the technical user interface (TUI). The floodplain model generates water level conditions which are used as inputs to the fish habitat, vegetation and waterbird models. The results from each model are stored in their own database. The model results are then extracted by the an eco-health scoring algorithm (Young et al., 1999a,b) to calculate final scores for various combinations of localities and models which are viewed within the PUI.

The system has been designed to be generic in structure. The underlying RAISON DSS is written in Visual Basic and $\mathrm{C} / \mathrm{C}++$ programming languages 
and is modular in nature. It provides a generic framework that allows integration of numerical and textual data, raster and vector maps and knowledge in the form of rule bases.

The system consists of many components, including a relational database management system that works with SQL, raster and vector based geographical information system (GIS), graphing module (that produces standard graphs as well as time-series plots), statistics package (that performs basic statistics, parametric and non-parametric tests and time series analysis) and a rule based expert system that can handle both forward and backward chaining logic. RAISON also includes an extensive library of functions that are implemented through Dynamic Data Exchange (DDE) and Dynamic Linked Libraries (DLL) and that are used for communicating with the RAISON system. These functions can be called from external applications or programs that are independent of the RAISON software. For example, the RAISON expert system itself uses these functions to display the results of an expert system run onto the RAISON map.

\section{ECOLOGICAL MODELLING APPROACH AND IMPLEMENTATION}

In general, there were three possible ways of implementing the ecological models within the decision support system. The first way was to write computer code for the individual models based upon basic scientific principles and known relationships of water depth, plant growth, etc. A second way was to write each ecological model as a rule-base, using optimal decision tree algorithms, with if-then rules that accept numeric values, and qualitative descriptions or fuzzy membership functions for the attributes. The third way was to re-structure the rule base and use other artificial intelligence algorithms such as the frame-and-object approach. In the end, despite attempts to use the expert system approaches, we were forced to use the first method as the level of knowledge available for the ecosystems within the Murray-Darling Basin was found to be quite limited. The following is a brief description of the individual models.

The ability to model the movement of water between the instream and floodplain is required for assessing the quality of aquatic habitat for riverine biota including fish, vegetation and waterbirds. The floodplain model employed here has been developed for modelling the average water depth and flood duration using a partial water balance. The floodplain is modelled as a set of storages which fill and empty over time using the daily flows. The outputs are used to predict the habitat conditions for waterbirds and floodplain vegetation. 
An index-based fish habitat model is used which contains separate components for adult survival and for breeding success. These two components are applied to different groupings of potamodromous native fish. Index values from the two components give an overall indication of fish habitat conditions. Final quantitative output is 'Fish habitat index', with one value per year per species group. Additional output is provided in a text report summarizing river section /flow scenario parameters with respect to fish habitat. Insufficient knowledge of fish life histories and environmental data prevent individual-based modelling of fish responses and the development of population-based models.

The algal model is used to predict when a weir pool environment will stratify. This is based upon the location, and physical aspects of the weir, river flow, wind speed, air temperature, relative humidity, water turbidity and time of year. The model presents information for daily algal cell counts, daily warning levels for algal bloom conditions and summary information about various time scales for different flow conditions. This information is intended to give an indication of the overall likelihood of nuisance blooms for different flow management options.

The vegetation model for the EFDSS considers those parts of the ecosystem which are affected directly by changes in the instream and overbank flow regimes due to regulation. Consequently, the riparian and floodplain vegetation are only included in the model. The model considers two main cycles that vegetation communities pass through, namely regeneration and maintenance. Each has influencing variables which are represented by a response function, indicating an indexed value ranging between 0 and 1 . Each vegetation stage is assessed annually, where the index value for each stage is determined as the highest value achieved for the year.

The breeding success of many migratory waterbird species is closely linked with floodplain conditions, most importantly emergent and submerged vegetation. The index-based waterbird model uses the results from the floodplain model, specifically duration of flood, depth, rate of rise, and flood variation to evaluate the quality of habitat for breeding and the likelihood of success.

The ecology-hydrology indices model calculates a set of flow indices based on the magnitude, mean, variations, duration and timing of flow extremes (drought and flooding), and flow change patterns, that may affect the river basin ecology. 


\section{TECHNICAL USER INTERFACE (TUI)}

The technical user interface (TUI) allows the EFDSS user direct access to the databases and models used within the system. It is intended to be used by experienced ecological modellers. An initial TUI interface allows the user to select which model they wish to run. It is by nature a native Windows interface more familiar to a skilled computer user rather than a more graphical/pictorial approach which is used in the public user interface (PUI) described below. An example of a TUI interface is shown in Figure 1 for the waterbird model. The TUI allows for importing of basic data for setting up the model parameters. A data loader is used to provide the daily IQQM flow data in the appropriate format for each of the models. It performs various analyses on the data and stores the processed flow data into the databases and lookup tables. When new IQQM scenarios are run, the daily flow data produced are loaded via the data loader without affecting the data already loaded.

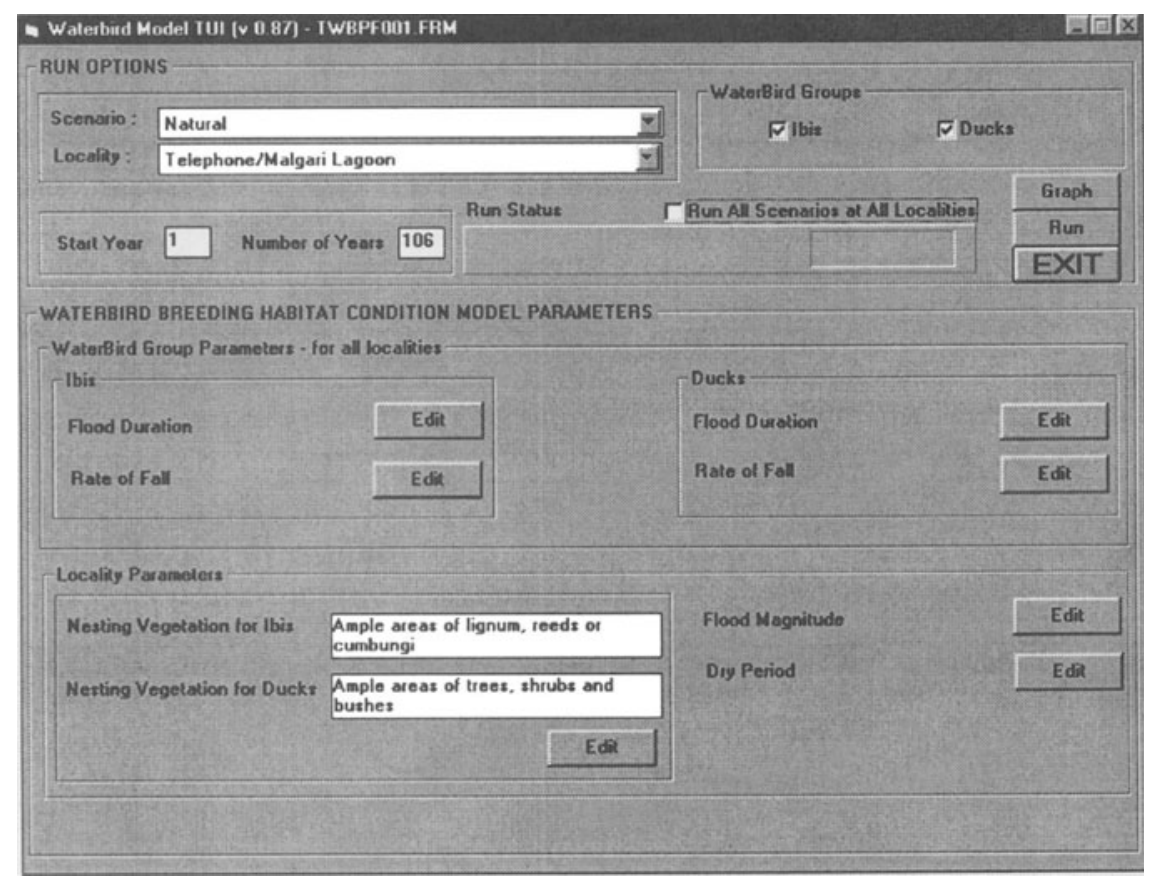

Figure 1. Waterbird model technical user interface main startup form.

In the EFDSS TUI-integrated modelling approach, a scenario is a combination of the sets of data and input parameters for all models (including IQQM). This means that each scenario will be composed of a 
definite set of data and input parameters to describe the IQQM, floodplain , fish, waterbird, vegetation and hydrology/ecology indices models, as well as the integrated relationship between models (spatial and temporal). This integrated modelling approach allows the user to add new scenarios for the fish model for example. A model dependency look-up table is used by the system to indicate which other models have to be rerun to complete the new EFDSS scenario. This modular approach allows the TUI user to test various combinations of sub-scenarios in an efficient manner.

\section{PUBLIC USER INTERFACE (PUI)}

The conceptual structure of the PUI is represented in the home screen (Figure 2), which groups the EFDSS assessment modules into four categories: (i) physical condition assessments, (ii) habitat condition assessments, (iii) nuisance assessments, and (iv) integrated assessments. These categories apply to any EFDSS application; however, the actual assessment modules in each category may vary between applications. The first category - physical condition assessments, includes tools to investigate the river and floodplain hydrology data in a range of graphic and tabular ways.

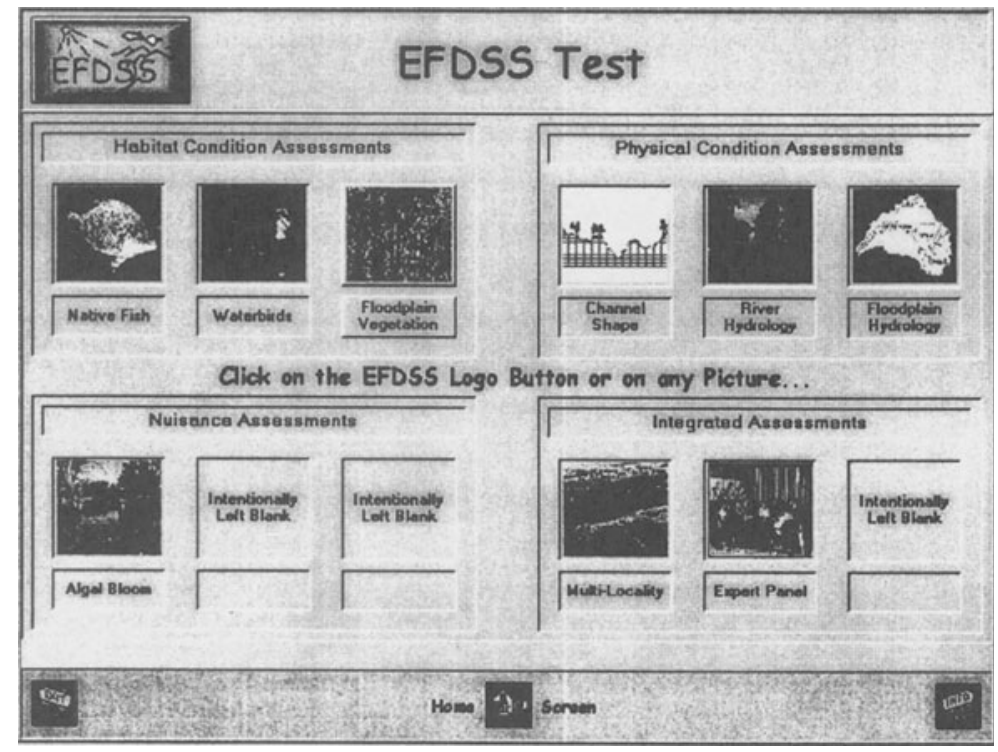

Figure 2. Public User Interface (PUI) home page.

This category also includes a module which gives qualitative assessments of channel shape change. The second and third categories - habitat condition 
and nuisance assessments - include modules which respectively, provide assessments of the positive and negative ecological aspects of condition of the riverine environment. In the habitat condition category of the prototype EFDSS application, there are modules for native fish, floodplain vegetation, and waterbird breeding habitat condition. In the nuisance category of the prototype application, there is a single module for algal bloom assessments. The final category - integrated assessments - includes modules that provide assessments which are integrated across space and time. In the prototype, two such modules are provided: a multi-locality integrated assessment module (MIA), and an expert panel assessment module (XPA). The MIA integrates the outputs from the habitat condition and nuisance assessment modules, with the former treated as positive values, and the latter as negative values. The MIA integrates across localities and river zones, and across time. The XPA provides an independent and qualitative assessment for an entire zone, for a whole scenario, based on expert knowledge of the affects of different types of hydrological change.

\section{SCENARIO RESULTS}

In Figure 4 a comparison is presented of a habitat annual condition assessment for native fish - group 1 (perch) for natural versus current flow scenarios at MacIntyre Brook over a 100 year period. In this case it can very easily be seen that the natural scenario provides much better habitat conditions over the simulation period. It also shows that neither good or excellent conditions are provided under either scenario over the period of simulation.

\section{CONCLUDING REMARKS}

The design concepts of the EFDSS have changed and evolved over the years since it was originally conceived (Young et al., 1997). One could design the system from the top down or from the bottom up. For this project, we are required to follow a well established protocol that it should have feedback from the stakeholders and hence the project should be designed from the bottom up. This resulted in the PUI component of the system receiving the bulk of the design considerations and programming efforts. 


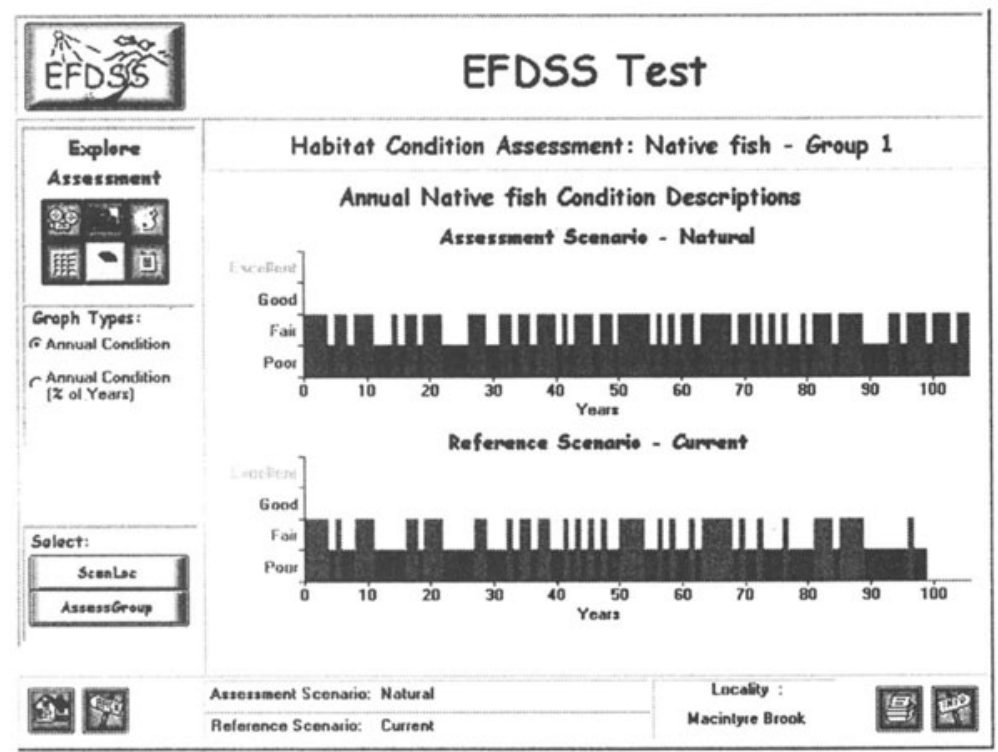

Figure 4. Native Fish Habitat Condition Assessment for Natural and Current Scenarios at McIntyre Brook

Quite a large portion of the twenty months of work was spent on communication among the team members, scientists, policy advisors and potential users. For future development, efforts should be devoted to model verification and uncertainty analysis.

\section{ACKNOWLEDGEMENTS}

The authors thank the Murray-Darling Basin Commission, the Land and Water Resources Research and Development Corporation, and Environment Australia for financial support of this project. We wish to thank all of the project staff from CSIRO and NWRI who contributed both technically and intellectually to this project, including Frances Marston, Alex Storey, Philip Fong, and Craig McCrimmon. Special thanks are also due to Dr. David Swayne for support and encouragement to write this paper. 


\section{REFERENCES}

Lam, D.C.L., C.I. Mayfield, D.A. Swayne and K. Hopkins, 1994. A prototype information system for watershed management and planning. J. Biological Systems, 2(4):499-517.

Podger, G.M., Sharma, P.K., and Black, D.C. 1994. An integrated water quantity and quality modelling suite. Proceedings of Environmental Flows Seminar, AWWA, Australia.

Young, W.J., Booty, W.G., Whigham, P.A., and Lam, D.C.L. 1999a. Integrated assessments of river health using decision support software. In: Proceedings of ISESS 1999 Third International Symposium on Environmental Software Systems, University of Otago, Dunedin, New Zealand.

Young, W.J., Booty, W.G., Cuddy, S.M., Farely, T.F., Lam, D.C.L. and Whigham, P.A. 1999b. Ecological and hydrological assessments of environmental flow regimes using decision support software. Proceedings of an International Conference: Assessing the Ecological Integrity of Running Waters, Vienna, 1998. [In press].

Young, W.J., Lam, D.C., Ressel, V., and Wong, I.W. 1997. Development of an Environmental Flows Decision Support System. In: Denzer, R., Swayne, D., and Schimak, G. (Eds). Environmental Software Systems, Vol. 2, Proceedings of International Symposium on Environmental Software Systems, pp 87-94, Chapman \& Hall, London. 